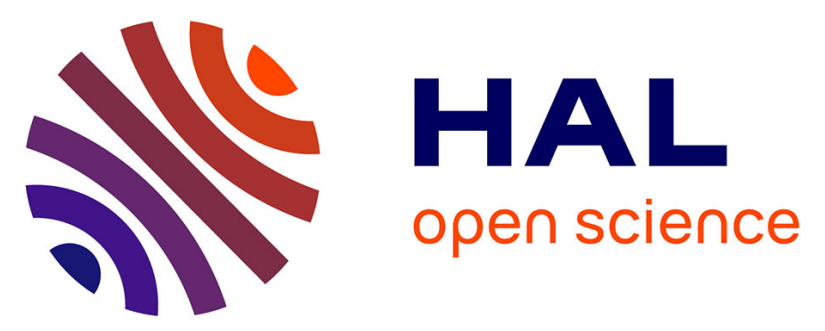

\title{
State-dependent implicit sweeping process in the framework of quasistatic evolution quasi-variational inequalities
}

\author{
Samir Adly, Tahar Haddad, Le Ba Khiet
}

\section{- To cite this version:}

Samir Adly, Tahar Haddad, Le Ba Khiet. State-dependent implicit sweeping process in the framework of quasistatic evolution quasi-variational inequalities. Journal of Optimization Theory and Applications, 2019, 182 (2), pp.473-493. 10.1007/s10957-018-1427-x . hal-02386012

\section{HAL Id: hal-02386012 \\ https://hal.science/hal-02386012}

Submitted on 14 Jan 2020

HAL is a multi-disciplinary open access archive for the deposit and dissemination of scientific research documents, whether they are published or not. The documents may come from teaching and research institutions in France or abroad, or from public or private research centers.
L'archive ouverte pluridisciplinaire HAL, est destinée au dépôt et à la diffusion de documents scientifiques de niveau recherche, publiés ou non, émanant des établissements d'enseignement et de recherche français ou étrangers, des laboratoires publics ou privés. 


\title{
State-Dependent Implicit Sweeping Process in the Framework of Quasistatic Evolution Quasi-Variational Inequalities
}

\author{
Samir Adly ${ }^{1}$ (D) Tahar Haddad ${ }^{2} \cdot$ Ba Khiet Le $^{3}$
}

\begin{abstract}
This paper deals with the existence and uniqueness of solutions for a class of statedependent sweeping processes with constrained velocity in Hilbert spaces without using any compactness assumption, which is known to be an open problem. To overcome the difficulty, we introduce a new notion called hypomonotonicity-like of the normal cone to the moving set, which is satisfied by many important cases. Combining this latter notion with an adapted Moreau's catching-up algorithm and a Cauchy technique, we obtain the strong convergence of approximate solutions to the unique solution, which is a fundamental property. Using standard tools from convex analysis, we show the equivalence between this implicit state-dependent sweeping processes and quasistatic evolution quasi-variational inequalities. As an application, we study the state-dependent quasistatic frictional contact problem involving viscoelastic materials with short memory in contact mechanics.
\end{abstract}

Keywords Moreau's sweeping process · Evolution variational inequalities · Unilateral constraints · Quasistatic frictional contact problems

Mathematics Subject Classification 49J40 - 47J20 - 47J22 · 58E35 · 74M15 - 74M10 • $74 \mathrm{G} 25$

Samir Adly

samir.adly@unilim.fr

Tahar Haddad

haddadtr2000@yahoo.fr

Ba Khiet Le

lebakhiet@gmail.com

1 XLIM UMR-CNRS 7252, Université de Limoges, 87060 Limoges, France

2 Laboratoire LMPEA, Université de Jijel, 18000 Jijel, Algeria

3 Instituto de Ciencias de la Ingeniería, Universidad de O’Higgins, Rancagua, Chile 


\section{Introduction}

In the seventies, J. J. Moreau introduced and thoroughly studied the so-called sweeping processes [1-3], which have a particular form of differential inclusions (5) involving the outward normal cone to a moving closed and convex set in a Hilbert space. The original motivation is to model quasistatic evolution in elastoplasticity, friction dynamics, granular material, contact dynamics. However, many applications of the sweeping processes can be also found nowadays in nonsmooth mechanics, convex optimization, modeling of crowd motion, mathematical economics, dynamic networks, switched electrical circuits, etc (see, e.g., [4-8] and the references therein). Existence and uniqueness of solutions of such systems and their classical variants (subjected to perturbation forces, state-dependent, second order sweeping processes, etc) have been studied fruitfully in the literature (see, e.g., [2,3,6,8-11]).

Recently in [6], the authors proposed a new variant with velocity in the constraint (6) and provided various applications in electrical circuits. Under the coercivity of the bounded symmetric linear monotone involved operators and the Lipschitz continuity of the moving set (assumed to be bounded) with respect to the Hausdorff distance, the authors showed in [6] an existence and uniqueness of solution of problem (6). Later the case of unbounded moving set is relaxed in [12], by assuming only the semicoercivity of the involved linear operators. Continuing the idea, in [13] the authors showed the existence and uniqueness of solutions for a new variant (7), by taking into account a linear combination of the velocity and state in Hilbert spaces. As an application, the quasistatic antiplane frictional contact problem for linear elastic materials with short memory was formulated as an implicit sweeping process with velocity constraint. This work creates a bridge between the different mathematical abstract results in the literature and their applications in nonsmooth mechanics, which was the initial motivations of Moreau [1].

In this paper, we are interested in a generalization of the variant studied in [13] to the case of a state-dependent moving set (11) without using any compactness assumption, which is known to be an open problem. The additional initial viability condition (8) is no longer required here, which allows nonzero initial velocity. The set of constraints is supposed to move in an absolutely continuous way with respect to time and Lipschitz continuous with respect to the state. The normal cone to the moving set is assumed to be hypomonotone-like (see Assumption 2 for the definition). Let us mention that the class of set-valued maps satisfying the latter property is very large for applications (see Lemmas 3.1, 3.2 for more details).

To conclude this introduction, we mention that the present work can be considered as an improvement and an extension of the research initiated by the authors in [13]. In fact, the technique utilized in the current paper differs from the one used in [13]. The proof here is based on an implicit time discretization combined with the construction of a Cauchy sequence of functions via linear interpolation that converges strongly to a unique solution of (11). Clearly, the uniqueness of a solution as well as the strong convergence of approximate solutions to the unique solution, obtained in this paper, are important properties, compared to the weak convergence of a subsequence obtained in $[6,12,13]$. Note that the compatibility condition (8) is no longer necessary here, 
which is in concordance with the result obtained in [14] for the quasistatic antiplane frictional contact problem (see Theorem 10.3 page 193 and Remark 4.6 in [13]).

The paper is organized as follows. We recall in Sect. 2 some basic notation, definitions and results which are used throughout the paper. In Sect. 3, the well-posedness of (11) is thoroughly studied by using an implicit time discretization. An application to the quasistatic frictional contact problem is given in Sect. 4. Finally, Sect. 5 closes the paper with some concluding remarks.

\section{Notation, Preliminaries and General Framework}

We begin with some notation used in the paper. Let $H$ be a Hilbert space. Denote by $\|\cdot\|$ the norm in $H$, by $\langle\cdot, \cdot\rangle$ the scalar product and by $\mathbb{B}$ the closed unit ball in $H$. Let be given a closed, convex set $C \subset H$. The distance from a point $s$ to $C$ is defined by

$$
d(s, C):=\inf _{x \in C}\|s-x\| .
$$

The Hausdorff distance between two closed, convex sets $C_{1}, C_{2} \subset H$ is defined by

$$
d_{H}\left(C_{1}, C_{2}\right):=\max \left\{\sup _{x_{1} \in C_{1}} d\left(x_{1}, C_{2}\right), \sup _{x_{2} \in C_{2}} d\left(x_{2}, C_{1}\right)\right\}
$$

It is easy to see that

$$
d_{H}\left(C_{1}, C_{2}\right)=\varepsilon \Rightarrow C_{1} \subset C_{2}+\varepsilon \mathbb{B} \text { and } C_{2} \subset C_{1}+\varepsilon \mathbb{B} .
$$

The normal cone of a closed and convex set $S$ is defined as follows

$$
N_{S}(x):=\left\{x^{*} \in H:\left\langle x^{*}, y-x\right\rangle \leq 0, \forall y \in S\right\} .
$$

It is not difficult to see that

$$
x^{*} \in N_{S}(x) \Leftrightarrow \sigma\left(S, x^{*}\right)=\left\langle x^{*}, x\right\rangle \text { and } x \in S,
$$

where $\sigma$ is the support function, defined by

$$
\sigma\left(S, x^{*}\right):=\sup _{\xi \in S}\left\langle x^{*}, \xi\right\rangle, \quad x^{*} \in H .
$$

Given a continuous function $v: \mathbb{R} \rightarrow \mathbb{R}$, the modulus of continuity of $v$ is the function $\omega:[0,+\infty] \rightarrow[0,+\infty]$, defined by

$$
\omega(h):=\sup _{|x-y| \leq h}|v(x)-v(y)|, \quad h>0 .
$$

In the following, we summarize some known definitions and results concerning maximally monotone operators. The domain and graph of a set-valued operator $T: H \rightrightarrows H$ are defined, respectively, by 


$$
D(T):=\{x \in H: T(x) \neq \emptyset\}, \quad \operatorname{gph}(T):=\{(x, y): x \in H, y \in T(x)\} .
$$

The inverse of $T$ is defined by

$$
T^{-1}\left(x^{*}\right):=\left\{x \in H: x^{*} \in T(x)\right\}, x^{*} \in H .
$$

The operator $T$ is said to be monotone, if for all $x, y \in H, x^{*} \in T(x), y^{*} \in T(y)$, we have

$$
\left\langle x^{*}-y^{*}, x-y\right\rangle \geq 0 \text {. }
$$

In addition, if there is no monotone mapping $T^{\prime}$ such that $\operatorname{gph}(T)$ is contained strictly in $\operatorname{gph}\left(T^{\prime}\right)$, then $T$ is called maximally monotone.

The operator $T$ is said to be coercive, if for all $\left(x_{n}, y_{n}\right) \in \operatorname{gph}(T)$ such that $\lim _{n \rightarrow+\infty}\left\|x_{n}\right\|=+\infty$, we have

$$
\lim _{n \rightarrow+\infty} \frac{\left\langle x_{n}-x_{0}, y_{n}\right\rangle}{\left\|x_{n}\right\|}=+\infty
$$

for some $x_{0} \in H$. It is known that coercive maximally monotone operators in reflexive Banach spaces are surjective (see, e.g, [17]). We close this section with some versions of discrete and continuous Gronwall's inequalities [18].

Lemma 2.1 Let $\alpha>0$ and $\left(u_{n}\right),\left(\beta_{n}\right)$ be nonnegative sequences satisfying

$$
u_{n} \leq \alpha+\sum_{k=0}^{n-1} \beta_{k} u_{k} \quad \forall n=0,1,2, \ldots \quad\left(\text { with } \beta_{-1}:=0\right)
$$

Then, for all $n$, we have

$$
u_{n} \leq \alpha \exp \left(\sum_{k=0}^{n-1} \beta_{k}\right)
$$

Lemma 2.2 Let $T>0$ be given and $a(\cdot), b(\cdot) \in L^{1}([0, T] ; \mathbb{R})$ with $b(t) \geq 0$ for almost all $t \in[0, T]$. Let the absolutely continuous function $w:[0, T] \rightarrow \mathbb{R}_{+}$satisfy

$$
(1-\alpha) w^{\prime}(t) \leq a(t) w(t)+b(t) w^{\alpha}(t), \quad \text { a.e. } t \in[0, T],
$$

where $0 \leq \alpha<1$. Then for all $t \in[0, T]$, one has

$$
w^{1-\alpha}(t) \leq w^{1-\alpha}(0) \exp \left(\int_{0}^{t} a(\tau) \mathrm{d} \tau\right)+\int_{0}^{t} \exp \left(\int_{s}^{t} a(\tau) \mathrm{d} \tau\right) b(s) \mathrm{d} s .
$$

The mathematical formulation of the sweeping process introduced by J.J. Moreau is given by 


$$
\begin{aligned}
& \dot{x}(t) \in-N_{C(t)}(x(t)) \quad \text { a.e. } t \in[0, T], \\
& x(0)=x_{0} \in C(0),
\end{aligned}
$$

where $N_{C(t)}(\cdot)$ denotes the normal cone operator associated to the closed and convex set $C(t)$ in a Hilbert space $H$.

In [6], the authors studied the following new variant

$$
\begin{aligned}
& A_{1} \dot{u}(t)+A_{0} u(t)-f(t) \in-\mathrm{N}_{C(t)}(\dot{u}(t)) \text { a.e. } t \in[0, T], \\
& u(0)=u_{0},
\end{aligned}
$$

where $C(\cdot)$ is a bounded moving set, $A_{1}, A_{0}: H \rightarrow H$ are two bounded, symmetric, linear and monotone operators and $f:[0, T] \rightarrow H$ is a continuous mapping. In [13], the authors proposed the following model

$$
\begin{aligned}
& \dot{u}(t) \in-\mathrm{N}_{C(t)}(A \dot{u}(t)+B u(t)) \quad \text { a.e. } t \in[0, T], \\
& u(0)=u_{0},
\end{aligned}
$$

with additional initial condition

$$
B u_{0} \in C(0)
$$

where $A, B: H \rightarrow H$ are two linear, bounded, symmetric and monotone operators and $A$ is coercive, i.e., there exists some $\beta>0$ such that

$$
\langle A x, x\rangle \geq \beta\|x\|^{2}, \quad \forall x \in H .
$$

The moving set $t \mapsto C(t)$ varies in an absolutely continuous way, i.e., there exists some absolutely continuous function $v:[0, T] \rightarrow \mathbb{R}$ such that

$$
d_{H}(C(s), C(t)) \leq|v(s)-v(t)| \text { for all } s, t \in[0, T]
$$

where $d_{H}$ denotes the Hausdorff distance.

In this paper, we are interested in a generalization of the variant studied in [13] to the case of a state-dependent moving set without requiring the additional initial viability condition, which allows nonzero initial velocity. More precisely, let $A, B: H \rightarrow H$ be two linear, bounded and symmetric operators with coercive $A$ and $C:[0, T] \times H \rightrightarrows H$ be a set-valued mapping with nonempty, closed and convex values. Our aim is to study the well-posedness of the following sweeping process

$$
\begin{aligned}
& \dot{u}(t) \in-\mathrm{N}_{C(t, u(t))}(A \dot{u}(t)+B u(t)) \text { a.e. } t \in[0, T], \\
& u(0)=u_{0},
\end{aligned}
$$

without requiring the additional initial viability condition $B u_{0} \in C\left(0, u_{0}\right)$, which allows nonzero initial velocity. 


\section{Main Result}

In this section, the well-posedness (in the sense of existence and uniqueness of solutions) of the state-dependent sweeping processes in Hilbert spaces is studied. Note that the generalization for reflexive Banach spaces can be done in a similar way. First, let us make the following assumptions.

Assumption 1 The set-valued mapping $C:[0, T] \times H \rightrightarrows H$ has nonempty, closed and convex values and there exists an absolutely continuous function $v:[0, T] \rightarrow \mathbb{R}$ and $L \geq 0$ such that for all $s, t \in[0, T]$ and $u, w \in H$

$$
d_{H}(C(s, u), C(t, w)) \leq|v(s)-v(t)|+L\|u-w\| .
$$

Assumption 2 The normal cone of $C$ is hypomonotone-like, in the sense that for given $R>0$, there exist $\tilde{L} \geq 0$ and an absolutely continuous function $\tilde{v}:[0, T] \rightarrow \mathbb{R}$ such that if

$$
a_{i} \in N_{C\left(t_{i}, u_{i}\right)}\left(b_{i}\right) \text { for } a_{i} \in H, u_{i}, b_{i} \in R \mathbb{B}, t_{i} \in[0, T], i=1,2
$$

then

$$
\left\langle a_{1}-a_{2}, b_{1}-b_{2}\right\rangle \geq-\tilde{L}\left\|a_{1}-a_{2}\right\|\left\|u_{1}-u_{2}\right\|-\left(\left\|a_{1}\right\|+\left\|a_{2}\right\|\right)\left|\tilde{v}\left(t_{1}\right)-\tilde{v}\left(t_{2}\right)\right| .
$$

Assumption 3 Let A, B:H $\rightarrow H$ be symmetric, linear and bounded operators satisfying

$$
\langle A x, x\rangle \geq \beta\|x\|^{2} \text { and }\langle B x, x\rangle \geq 0 \text { for some } \beta>0 .
$$

Remark 3.1 If $C(t, u) \equiv C$ is a constant, closed and convex set, then the normal cone of $C$ is monotone and obviously is hypomonotone-like. Now we provide some important non-constant cases to show that the class of the moving set $C$ satisfying Assumptions 1 and 2 is large for applications.

Lemma 3.1 Let $C(t, u)=f(t, u)+C_{1}(t)$ for all $t \in[0, T], x \in H$, where $C_{1}:[0, T] \rightrightarrows H$ has nonempty, closed and convex values, $v_{1}$-absolutely continuous and $f:[0, T] \times H \rightarrow H$ is $v_{2}$-absolutely continuous with respect to time, L-Lipschitz continuous with respect to state, i.e., there exist absolutely continuous functions $v_{1}, v_{2}:[0, T] \rightarrow \mathbb{R}$ and $L \geq 0$ such that

$$
\begin{aligned}
& d_{H}\left(C_{1}(t), C_{1}(s)\right) \leq\left|v_{1}(t)-v_{1}(s)\right| \text { and } \\
& \|f(t, u)-f(s, w)\| \leq\left|v_{2}(t)-v_{2}(s)\right|+L\|u-w\|,
\end{aligned}
$$

for all $t, s \in[0, T]$ and $u, w \in H$. Then Assumptions 1 and 2 are satisfied.

Proof It is easy to see that $C$ has nonempty, closed and convex values and $d_{H}(C(s, u), C(t, w)) \leq|v(s)-v(t)|+L\|u-w\|$, for all $s, t \in[0, T], u, w \in H$, 
where $v(t):=\int_{0}^{t}\left(\left|\dot{v}_{1}(s)\right|+\left|\dot{v}_{2}(s)\right|\right) \mathrm{d} s$. To check the hypomonotonicity-like of the normal cone of $C$, let us note that

$$
a_{i} \in N_{C\left(t_{i}, u_{i}\right)}\left(b_{i}\right)=\mathrm{N}_{C_{1}\left(t_{i}\right)+f\left(t_{i}, u_{i}\right)}\left(b_{i}\right)=N_{C_{1}\left(t_{i}\right)}\left(b_{i}-f\left(t_{i}, u_{i}\right)\right), \quad i=1,2 .
$$

Hence

$$
\left\langle a_{1}, z-b_{1}+f\left(t_{1}, u_{1}\right)\right\rangle \leq 0, \text { for all } z \in C_{1}\left(t_{1}\right) .
$$

In addition, one has $b_{2} \in C_{1}\left(t_{2}\right)+f\left(t_{2}, u_{2}\right) \subset C_{1}\left(t_{1}\right)+\left|v_{1}\left(t_{1}\right)-v_{1}\left(t_{2}\right)\right| \mathbb{B}+f\left(t_{2}, u_{2}\right)$. Combining with the last inequality, we obtain

$$
\left\langle a_{1}, b_{2}-b_{1}+f\left(t_{1}, u_{1}\right)-f\left(t_{2}, u_{2}\right)\right\rangle \leq\left|v_{1}\left(t_{1}\right)-v_{1}\left(t_{2}\right)\right|\left\|a_{1}\right\| .
$$

Similarly, one has

$$
\left\langle a_{2}, b_{1}-b_{2}+f\left(t_{2}, u_{2}\right)-f\left(t_{1}, u_{1}\right)\right\rangle \leq\left|v_{1}\left(t_{1}\right)-v_{1}\left(t_{2}\right)\right|\left\|a_{2}\right\| .
$$

From (17) and (18), we deduce that

$$
\begin{aligned}
\Psi & =\left\langle a_{1}-a_{2}, b_{1}-b_{2}\right\rangle \\
& \geq\left\langle a_{1}-a_{2}, f\left(t_{1}, u_{1}\right)-f\left(t_{2}, u_{2}\right)\right\rangle-\left(\left|v_{1}\left(t_{1}\right)-v_{1}\left(t_{2}\right)\right|\right)\left(\left\|a_{1}\right\|+\left\|a_{2}\right\|\right) \\
& \geq-L\left\|a_{1}-a_{2}\right\|\left\|u_{1}-u_{2}\right\|-\left(\left|v_{1}\left(t_{1}\right)-v_{1}\left(t_{2}\right)\right|+\left(\left|v_{2}\left(t_{1}\right)-v_{2}\left(t_{2}\right)\right|\right)\left(\left\|a_{1}\right\|+\left\|a_{2}\right\|\right)\right. \\
& \geq-L\left\|a_{1}-a_{2}\right\|\left\|u_{1}-u_{2}\right\|-\left(\left|v\left(t_{1}\right)-v\left(t_{2}\right)\right|\right)\left(\left\|a_{1}\right\|+\left\|a_{2}\right\|\right),
\end{aligned}
$$

which completes the proof of Lemma 3.1.

Lemma 3.2 Let $C(t, u)=f_{1}(t, u)+f_{2}(t, u) \mathbb{B}$, where $f_{1}:[0, T] \times H \rightarrow H, f_{2}$ : $[0, T] \times H \rightarrow[\alpha,+\infty),(t, u) \mapsto f_{i}(t, u)$ is supposed to be $v_{i}$-absolutely continuous in $t, L_{i}$-Lipschitz continuous in u for some $L_{i} \geq 0, i=1,2$ and for some $\alpha>0$. Then Assumptions 1 and 2 are satisfied.

Proof Clearly $C$ has nonempty, closed and convex values and $d_{H}(C(s, u), C(t, w)) \leq|v(s)-v(t)|+L\|u-w\|$, for all $s, t \in[0, T], u, w \in H$, where $v(t):=\int_{0}^{t}\left(\left|\dot{v}_{1}(s)\right|+\left|\dot{v}_{2}(s)\right|\right) \mathrm{d} s$ and $L=L_{1}+L_{2}$. It remains to check the hypomonotonicity-like property. Let be given $R>0$ and

$$
a_{i} \in N_{f_{1}\left(t_{i}, u_{i}\right)+f_{2}\left(t_{i}, u_{i}\right) \mathbb{B}}\left(b_{i}\right)=\mathrm{N}_{f_{2}\left(t_{i}, u_{i}\right) \mathbb{B}}\left(b_{i}-f_{1}\left(t_{i}, u_{i}\right)\right), \quad b_{i}, u_{i} \in R \mathbb{B}, i=1,2 .
$$

Hence

$$
a_{1} \in N_{f_{2}\left(t_{1}, u_{1}\right) \mathbb{B}}\left(b_{1}-f_{1}\left(t_{1}, u_{1}\right)\right),
$$




$$
\begin{aligned}
a_{2} & \in N_{f_{2}\left(t_{2}, u_{2}\right) \mathbb{B}}\left(b_{2}-f_{1}\left(t_{2}, u_{2}\right)\right) \\
& =N_{f_{2}\left(t_{1}, u_{1}\right) \mathbb{B}}\left(\frac{\left(b_{2}-f_{1}\left(t_{2}, u_{2}\right)\right) f_{2}\left(t_{1}, u_{1}\right)}{f_{2}\left(t_{2}, u_{2}\right)}\right),
\end{aligned}
$$

where the second part follows from the fact that $f_{2}\left(t_{1}, u_{1}\right)>0, f_{2}\left(t_{2}, u_{2}\right)>0$ and the definition of the normal cone. From (19) and (20), we obtain

$$
\left\langle a_{1}-a_{2}, b_{1}-f_{1}\left(t_{1}, u_{1}\right)-\frac{\left(b_{2}-f_{1}\left(t_{2}, u_{2}\right)\right) f_{2}\left(t_{1}, u_{1}\right)}{f_{2}\left(t_{2}, u_{2}\right)}\right\rangle \geq 0,
$$

which implies that

$$
\left\langle a_{1}-a_{2}, b_{1}-f_{1}\left(t_{1}, u_{1}\right)\right\rangle \geq\left\langle a_{1}-a_{2}, \frac{\left(b_{2}-f_{1}\left(t_{2}, u_{2}\right)\right) f_{2}\left(t_{1}, u_{1}\right)}{f_{2}\left(t_{2}, u_{2}\right)}\right\rangle .
$$

Hence

$$
\begin{aligned}
& \begin{aligned}
\Phi & =\left\langle a_{1}-a_{2}, b_{1}-f_{1}\left(t_{1}, u_{1}\right)-b_{2}+f_{1}\left(t_{2}, u_{2}\right)\right\rangle \\
& \geq\left\langle a_{1}-a_{2}, \frac{\left(b_{2}-f_{1}\left(t_{2}, u_{2}\right)\right) f_{2}\left(t_{1}, u_{1}\right)}{f_{2}\left(t_{2}, u_{2}\right)}\right\rangle-\left\langle a_{1}-a_{2}, \frac{\left(b_{2}-f_{1}\left(t_{2}, u_{2}\right)\right) f_{2}\left(t_{2}, u_{2}\right)}{f_{2}\left(t_{2}, u_{2}\right)}\right\rangle \\
& =\left\langle a_{1}-a_{2}, \frac{\left(b_{2}-f_{1}\left(t_{2}, u_{2}\right)\right)\left(f_{2}\left(t_{1}, u_{1}\right)-f_{2}\left(t_{2}, u_{2}\right)\right)}{f_{2}\left(t_{2}, u_{2}\right)}\right\rangle \\
& \geq-k\left\|a_{1}-a_{2}\right\|\left\|f_{2}\left(t_{1}, u_{1}\right)-f_{2}\left(t_{2}, u_{2}\right)\right\|
\end{aligned} \\
& \text { where } k:=\frac{R+\sup _{t \in[0, T], u \in R \mathbb{B}}\left\|f_{1}(t, u)\right\|}{\alpha}<+\infty \text { Thus }
\end{aligned}
$$

$$
\begin{aligned}
\left\langle a_{1}-a_{2}, b_{1}-b_{2}\right\rangle & \geq-\left\|a_{1}-a_{2}\right\|\left(k\left\|f_{2}\left(t_{1}, u_{1}\right)-f_{2}\left(t_{2}, u_{2}\right)\right\|+\left\|f_{1}\left(t_{1}, u_{1}\right)-f_{1}\left(t_{2}, u_{2}\right)\right\|\right) \\
& \geq-\tilde{L}\left\|a_{1}-a_{2}\right\|\left\|u_{1}-u_{2}\right\|-\left(\left\|a_{1}\right\|+\left\|a_{2}\right\|\right)\left|\tilde{v}\left(t_{1}\right)-\tilde{v}\left(t_{2}\right)\right|,
\end{aligned}
$$

where $\tilde{L}:=L_{1}+k L_{2}$ and $\tilde{v}(t):=\int_{0}^{t}\left(\left|\dot{v}_{1}(s)\right|+k\left|\dot{v}_{2}(s)\right|\right) \mathrm{d} s$.

The following lemma is a consequence of a general chain rule concerning the composition of a convex function with a linear operator (see, e.g., [15, Theorem 4.13]). For this particular case, we can provide a simple and direct proof.

Lemma 3.3 Let Assumption 3 hold. Then for a given closed and convex set $D \subset H$, one has

$$
A N_{D}(A x)=N_{A^{-1}(D)}(x), \quad \forall x \in A^{-1}(D) .
$$

Proof For a given $x \in A^{-1}(D)$, we have

$$
\begin{aligned}
\zeta \in A N_{D}(A x) & \Leftrightarrow \exists \zeta^{\prime} \in N_{D}(A x) \text { s.t. } \zeta=A \zeta^{\prime} \\
& \Leftrightarrow \zeta=A \zeta^{\prime} \text { and }\left\langle\zeta^{\prime}, y-A x\right\rangle \leq 0, \quad \forall y \in D
\end{aligned}
$$




$$
\begin{aligned}
& \Leftrightarrow \zeta=A \zeta^{\prime} \text { and }\left\langle\zeta^{\prime}, A y^{\prime}-A x\right\rangle \leq 0, \quad \forall y^{\prime} \in A^{-1}(D) \\
& \Leftrightarrow\left\langle\zeta, y^{\prime}-x\right\rangle \leq 0, \quad \forall y^{\prime} \in A^{-1}(D) \\
& \Leftrightarrow \zeta \in N_{A^{-1}(D)}(x),
\end{aligned}
$$

and the proof is thereby completed.

The next result extends [16, Proposition 1] toward the infinite dimensional setting of this paper. Let us note that the surjectivity of the operator $N_{D}+A$ plays an important role in the well-posedness of the algorithm proposed in Theorem 3.1.

Lemma 3.4 Let Assumption 3 hold. Then for a given closed and convex set $D \subset H$, the set-valued mapping $x \mapsto N_{D}(x)+A x$ is surjective. In addition, the mapping $\left(N_{D}+A\right)^{-1}$ is single-valued and $1 / \beta$-Lipschitz continuous, with $\beta$ is the coercivity constant of $A$.

Proof Note that the set-valued mapping $N_{D}+A$ is maximally monotone and coercive. Hence, it is surjective (see, e.g, [17]). Let $x_{i} \in\left(N_{D}+A\right)^{-1}\left(y_{i}\right), i=1,2$. Then, one has $y_{i} \in N_{D}\left(x_{i}\right)+A x_{i}$ and thus

$$
\left\langle y_{2}-y_{1}, x_{2}-x_{1}\right\rangle \geq\left\langle A\left(x_{1}-x_{2}\right), x_{1}-x_{2}\right) \geq \beta\left\|x_{1}-x_{2}\right\|^{2},
$$

which implies that

$$
\left\|x_{1}-x_{2}\right\| \leq \frac{1}{\beta}\left\|y_{1}-y_{2}\right\| .
$$

Therefore, $\left(N_{D}+A\right)^{-1}$ is single-valued and $1 / \beta$-Lipschitz continuous.

Lemma 3.5 Assume Assumption 1. Then, there exists a constant $c>0$ which depends only on the initial data such that for all $t \in[0, T], u \in H$, we can find some $y \in C(t, u)$ satisfying $\|y\| \leq c+L\left\|u-u_{0}\right\|$.

Proof Fix some $y_{0} \in C\left(0, u_{0}\right)$. From Assumption 1, one has

$$
y_{0} \in C\left(0, u_{0}\right) \subset C(t, u)+\left(|v(t)-v(0)|+L\left\|u-u_{0}\right\|\right) \mathbb{B} .
$$

Hence, we can choose some $y \in C(t, u)$ such that

$$
\left\|y-y_{0}\right\| \leq|v(t)-v(0)|+L\left\|u-u_{0}\right\|
$$

By setting $c:=|v(0)|+\max _{t \in[0, T]}|v(t)|+\left\|y_{0}\right\|$, the conclusion follows.

Now, we are ready to state the main result.

Theorem 3.1 Assume Assumptions 1, 2 and 3 satisfied. Then problem (11) has a unique solution which is Lipschitz continuous. 
Proof Let be given some positive integer $n \geq 1$, define $h_{n}:=T / n$ and $t_{i}^{n}:=i h$ for $0 \leq i \leq n-1$. We approximate (11) by the following adapted Moreau's catching-up algorithm:

- Set $u_{0}^{n}=u_{0}$.

- For $0 \leq i \leq n-1$, we want to find $v_{i+1}^{n}$ and $u_{i+1}^{n}$ such that

$$
\begin{aligned}
& v_{i+1}^{n} \in-\mathrm{N}_{C\left(t_{i+1}^{n}, u_{i}^{n}\right)}\left(A v_{i+1}^{n}+B u_{i}^{n}\right), \\
& u_{i+1}^{n}=u_{i}^{n}+h_{n} v_{i+1}^{n} .
\end{aligned}
$$

The first line of (22) can be rewritten as follows

$$
v_{i+1}^{n} \in-N_{C\left(t_{i+1}^{n}, u_{i}^{n}\right)-B u_{i}^{n}}\left(A v_{i+1}^{n}\right),
$$

or equivalently by using Lemma 3.3,

$$
A v_{i+1}^{n} \in-A N_{D}\left(A v_{i+1}^{n}\right)=-\mathrm{N}_{A^{-1}(D)}\left(v_{i+1}^{n}\right),
$$

where $D=C\left(t_{i+1}^{n}, u_{i}^{n}\right)-B u_{i}^{n}$. Thanks to Lemma 3.4, one can compute uniquely $v_{i+1}^{n}$ by

$$
v_{i+1}^{n}=\left(N_{A^{-1}(D)}+A\right)^{-1}(0)
$$

and the algorithm is well defined.

(a) Boundedness of the iterates

From (22) and the coerciveness of $A$, one has, for all $v \in C\left(t_{i+1}^{n}, u_{i}^{n}\right)$, that

$$
\begin{aligned}
\beta\left\|v_{i+1}^{n}\right\|^{2} & \leq\left\langle A v_{i+1}^{n}, v_{i+1}^{n}\right\rangle \\
& \leq\left\langle A v_{i+1}^{n}+B u_{i}^{n}-v+v-B u_{i}^{n}, v_{i+1}^{n}\right\rangle \\
& \leq\left\langle v-B u_{i}^{n}, v_{i+1}^{n}\right\rangle \\
& =\left\|v-B u_{i}^{n}\right\|\left\|v_{i+1}^{n}\right\|
\end{aligned}
$$

which implies that

$$
\left\|v_{i+1}^{n}\right\| \leq \frac{1}{\beta}\left\|v-B u_{i}^{n}\right\|=\frac{1}{\beta}\left\|v-B u_{0}-h_{n} \sum_{k=1}^{i} B v_{k}^{n}\right\| .
$$

Using Lemma 3.5, we can always choose $v \in C\left(t_{i+1}^{n}, u_{i}^{n}\right)$ such that $\|v\| \leq c+L \| u_{i}^{n}-$ $u_{0}\left\|\leq c+h_{n} L \sum_{k=1}^{i}\right\| v_{k}^{n} \|$ for some constant $c>0$. The last inequality allows us to deduce

$$
\left\|v_{i+1}^{n}\right\| \leq \frac{1}{\beta}\left(c+\left\|B u_{0}\right\|\right)+\frac{1}{\beta} h_{n}(\|B\|+L) \sum_{k=1}^{i}\left\|v_{k}^{n}\right\| .
$$

By using a discrete version of Gronwall's inequality, one has

$$
\left\|v_{i+1}^{n}\right\| \leq \frac{1}{\beta}\left(c+\left\|B u_{0}\right\|\right) \exp \left(\frac{1}{\beta}(\|B\|+L) i h_{n}\right) .
$$


Consequently, the sequence $\left(v_{i}^{n}\right)_{1 \leq i \leq n}$ is uniformly bounded by

$$
M:=\frac{1}{\beta}\left(c+\left\|B u_{0}\right\|\right) \exp \left(\frac{1}{\beta}(\|B\|+L) T\right) .
$$

\section{(b) Convergence of the approximate solutions}

Now we construct the functions $u_{n}(\cdot), \theta_{n}(\cdot), \eta_{n}(\cdot)$ as follow: on $\left[t_{i}^{n}, t_{i+1}^{n}\right]$ for $0 \leq$ $i \leq n-1$, we set

$$
u_{n}(t):=u_{i}^{n}+\frac{u_{i+1}^{n}-u_{i}^{n}}{h_{n}}\left(t-t_{i}^{n}\right), \quad \theta_{n}(t):=t_{i}^{n}, \quad \eta_{n}(t):=t_{i+1}^{n} .
$$

Then, for all $t \in] t_{i}^{n}, t_{i+1}^{n}[$

$$
\dot{u}_{n}(t)=\frac{u_{i+1}^{n}-u_{i}^{n}}{h_{n}}=v_{i+1}^{n}, \quad \text { with } A v_{i+1}^{n}+B u_{i}^{n} \in C\left(t_{i+1}^{n}, u_{i}^{n}\right) \text { due to (22), }
$$

and

$$
\max \left\{\sup _{t \in[0, T]}\left|\theta_{n}(t)-t\right|, \sup _{t \in[0, T]}\left|\eta_{n}(t)-t\right|\right\} \leq h_{n} \rightarrow 0 \text { as } n \rightarrow+\infty .
$$

It is easy to see that $u_{n}(\cdot)$ is $M$-Lipschitz continuous with $u_{n}(0)=u_{0}$. Now let us prove that the sequence of functions $\left(u_{n}(\cdot)\right)_{n}$ is a Cauchy sequence in $C(0, T ; H)$. Let be given some positive integer $m, n$. Let $t \in] 0, T$ [ at which $u_{m}(\cdot)$ and $u_{n}(\cdot)$ are differentiable. There exist $i \in\{1, \ldots, m-1\}$ and $j \in\{1, \ldots, n-1\}$ such that $t \in\left[t_{i}^{m}, t_{i+1}^{m}\left[\right.\right.$ and $t \in\left[t_{j}^{n}, t_{j+1}^{n}\right.$ [, i.e., $\max \left\{\left|t-t_{i}^{m}\right|,\left|t-t_{i+1}^{m}\right|\right\} \leq h_{m}$ and $\max \{\mid t-$ $\left.t_{j}^{n}|| t-,t_{j+1}^{n} \mid\right\} \leq h_{n}$.

Note that $-v_{i+1}^{m} \in \mathrm{N}_{C\left(t_{i+1}^{m}, u_{i}^{m}\right)}\left(A v_{i+1}^{m}+B u_{i}^{m}\right)$ and $-v_{j+1}^{n} \in \mathrm{N}_{C\left(t_{j+1}^{n}, u_{j}^{n}\right)}\left(A v_{j+1}^{n}+\right.$ $\left.B u_{j}^{n}\right)$ and the sequences $\left(u_{i}^{m}\right)_{1 \leq i \leq m},\left(u_{j}^{n}\right)_{1 \leq j \leq n},\left(A v_{i+1}^{m}+B u_{i}^{m}\right)_{1 \leq i \leq m},\left(A v_{j+1}^{n}+\right.$ $\left.B u_{j}^{n}\right)_{1 \leq j \leq n}$ are uniformly bounded. From the hypomonotonicity-like of the normal cone of $C$ in Assumption 2, there exist $\tilde{L}>0$ and an absolutely continuous function $\tilde{v}:[0, T] \rightarrow \mathbb{R}$ such that

$$
\begin{aligned}
\Sigma & =\left\langle v_{j+1}^{n}-v_{i+1}^{m}, A v_{j+1}^{n}+B u_{j}^{n}-A v_{i+1}^{m}-B u_{i}^{m}\right\rangle \\
& =\left\langle v_{j+1}^{n}-v_{i+1}^{m}, A\left(v_{j+1}^{n}-v_{i+1}^{m}\right)\right\rangle+\left\langle v_{j+1}^{n}-v_{i+1}^{m}, B\left(u_{j}^{n}-u_{i}^{m}\right)\right\rangle \\
& \leq\left|\tilde{v}\left(t_{i+1}^{m}\right)-\tilde{v}\left(t_{j+1}^{n}\right)\right|\left(\left\|v_{i+1}^{m}\right\|+\left\|v_{j+1}^{n}\right\|\right)+\tilde{L}\left\|u_{i}^{m}-u_{j}^{n}\right\|\left\|v_{i+1}^{m}-v_{j+1}^{n}\right\| \\
& \leq\left|\tilde{v}\left(t_{i+1}^{m}\right)-\tilde{v}(t)+\tilde{v}(t)-\tilde{v}\left(t_{j+1}^{n}\right)\right|\left(\left\|v_{i+1}^{m}\right\|+\left\|v_{j+1}^{n}\right\|\right)+\tilde{L}\left\|u_{i}^{m}-u_{j}^{n}\right\|\left\|v_{i+1}^{m}-v_{j+1}^{n}\right\| \\
& \leq 2 M\left(\omega\left(h_{m}\right)+\omega\left(h_{n}\right)\right)+\tilde{L}\left\|u_{i}^{m}-u_{j}^{n}\right\|\left\|v_{i+1}^{m}-v_{j+1}^{n}\right\| \\
& =\varepsilon_{1}\left(h_{m}, h_{n}\right)+\tilde{L}\left\|u_{i}^{m}-u_{j}^{n}\right\|\left\|v_{i+1}^{m}-v_{j+1}^{n}\right\|
\end{aligned}
$$

where $\varepsilon_{1}\left(h_{m}, h_{n}\right):=2 M\left(\omega\left(h_{m}\right)+\omega\left(h_{n}\right)\right)$ and $\omega(\cdot)$ denotes the modulus of continuity of $\tilde{v}(\cdot)$. Using the coerciveness of $A$, the last inequality allows us to deduce that 


$$
\begin{aligned}
\Delta & =\frac{\beta}{2}\left\|v_{i+1}^{m}-v_{j+1}^{n}\right\|^{2} \\
& \leq \varepsilon_{1}\left(h_{m}, h_{n}\right)+(\tilde{L}+\|B\|)\left\|u_{i}^{m}-u_{j}^{n}\right\|\left\|v_{i+1}^{m}-v_{j+1}^{n}\right\|-\frac{\beta}{2}\left\|v_{i+1}^{m}-v_{j+1}^{n}\right\|^{2} \\
& \leq \varepsilon_{1}\left(h_{m}, h_{n}\right)+\frac{\tilde{L}_{1}^{2}}{2 \beta}\left\|u_{i}^{m}-u_{j}^{n}\right\|^{2} \quad \text { where } \tilde{L}_{1}=\tilde{L}+\|B\|
\end{aligned}
$$

(use the fact that $x \mapsto \alpha x-\beta x^{2}$ is concave and reaches its maximum at $x=\frac{\alpha}{2 \beta}$ )

$$
\begin{aligned}
& \leq \varepsilon_{1}\left(h_{m}, h_{n}\right)+\frac{\tilde{L}_{1}^{2}}{2 \beta}\left(\left\|u_{m}\left(\theta_{m}(t)\right)-u_{m}(t)+u_{m}(t)-u_{n}(t)+u_{n}(t)-u_{n}\left(\theta_{n}(t)\right)\right\|\right)^{2} \\
& \leq \varepsilon_{1}\left(h_{m}, h_{n}\right)+\frac{3 \tilde{L}_{1}^{2}}{2 \beta}\left(\left\|u_{m}\left(\theta_{m}(t)\right)-u_{m}(t)\right\|^{2}+\left\|u_{m}(t)-u_{n}(t)\right\|^{2}+\| u_{n}(t)\right. \\
& \left.\quad-u_{n}\left(\theta_{n}(t)\right) \|^{2}\right)
\end{aligned}
$$

(use the inequality $(a+b+c)^{2} \leq 3\left(a^{2}+b^{2}+c^{2}\right), \quad \forall a, b, c \in \mathbb{R}$ )

$$
\leq \varepsilon_{1}\left(h_{m}, h_{n}\right)+\frac{3 M^{2} \tilde{L}_{1}^{2}}{2 \beta}\left(h_{m}^{2}+h_{n}^{2}\right)+\frac{3 \tilde{L}_{1}^{2}}{2 \beta}\left\|u_{m}(t)-u_{n}(t)\right\|^{2} .
$$

Let

$$
\varepsilon_{2}\left(h_{m}, h_{n}\right):=\varepsilon_{1}\left(h_{m}, h_{n}\right)+\frac{3 M^{2}{\tilde{L_{1}}}^{2}}{2 \beta}\left(h_{m}^{2}+h_{n}^{2}\right)
$$

then $\varepsilon_{2}\left(h_{m}, h_{n}\right) \rightarrow 0$ as $m, n \rightarrow+\infty$ since $\omega\left(h_{n}\right) \rightarrow 0$ as $n \rightarrow+\infty$. Hence, from (30), we obtain

$$
\frac{\beta}{2}\left\|\dot{u}_{m}(t)-\dot{u}_{n}(t)\right\|^{2} \leq \varepsilon_{2}\left(h_{m}, h_{n}\right)+\frac{3 \tilde{L}_{1}^{2}}{2 \beta}\left\|u_{m}(t)-u_{n}(t)\right\|^{2}
$$

which implies that

$$
\left\|\dot{u}_{m}(t)-\dot{u}_{n}(t)\right\| \leq \varepsilon\left(h_{m}, h_{n}\right)+\frac{2 \tilde{L}_{1}}{\beta}\left\|u_{m}(t)-u_{n}(t)\right\|
$$

where

$$
\varepsilon\left(h_{m}, h_{n}\right):=\sqrt{\frac{2 \varepsilon_{2}\left(h_{m}, h_{n}\right)}{\beta}} .
$$

By using Gronwall's inequality, one has

$$
\left\|u_{m}(t)-u_{n}(t)\right\| \leq \exp \left(\frac{2 \tilde{L}_{1} T}{\beta}\right) \varepsilon\left(h_{m}, h_{n}\right) t \leq \exp \left(\frac{2 \tilde{L}_{1} T}{\beta}\right) \varepsilon\left(h_{m}, h_{n}\right) T
$$


Hence the sequence of functions $\left(u_{n}(\cdot)\right)_{n}$ is a Cauchy sequence in $C(0, T ; H)$ and there exists $u \in C(0, T ; H)$ such that $u_{n}$ converges to $u$ uniformly.

c) $u$ is a solution of $(11)$

Clearly, $u(0)=u_{0}$ and $u(\cdot)$ is $M$-Lipschitz continuous since for all $s, t \in[0, T]$, we have

$$
\|u(t)-u(s)\|=\lim _{n \rightarrow+\infty}\left\|u_{n}(t)-u_{n}(s)\right\| \leq M|t-s| .
$$

Consequently, $u(\cdot)$ is differentiable for almost every $t \in[0, T]$. By using BanachAlaoglu theorem, there exist some $\xi(\cdot)$ in $L^{2}(0, T ; H)$ and a subsequence, still denoted by $\left(\dot{u}_{n}(\cdot)\right)_{n}$ such that $\dot{u}_{n}(\cdot)$ converges weakly to $\xi(\cdot)$ in $L^{2}(0, T ; H)$.

Fix $t \in[0, T]$ and let $z(t):=u(t)-u_{0}-\int_{0}^{t} \xi(s) \mathrm{d} s$. We have

$$
\begin{aligned}
\left\langle z(t), u(t)-u_{0}-\int_{0}^{t} \xi(s) \mathrm{d} s\right\rangle & =\lim _{n \rightarrow+\infty}\left\langle z(t), u_{n}(t)-u_{0}-\int_{0}^{t} \xi(s) \mathrm{d} s\right\rangle \\
& =\lim _{n \rightarrow+\infty}\left\langle z(t), \int_{0}^{t}\left(\dot{u}_{n}(s)-\xi(s)\right) \mathrm{d} s\right\rangle \\
& =\lim _{n \rightarrow+\infty} \int_{0}^{T}\left\langle\mathbf{1}_{[0, t]}(s) z(t), \dot{u}_{n}(s)-\xi(s)\right\rangle \mathrm{d} s=0 .
\end{aligned}
$$

It implies that $u(t)=u_{0}+\int_{0}^{t} \xi(s) \mathrm{d} s$ for all $t \in[0, T]$ and hence $\dot{u}(t)=\xi(t)$ for almost every $t \in[0, T]$. Now, we prove that $A \dot{u}(t)+B u(t) \in C(t, u(t))$ for almost all $t \in[0, T]$. Given $\varepsilon>0$, let us define the set

$$
D_{\varepsilon}:=\left\{\varphi \in L^{2}(0, T ; H): A \varphi(t)+B u(t) \subset C(t, u(t))+\varepsilon \mathbb{B} \quad \text { a.e. } t \in[0, T]\right\} .
$$

It is easy to see that $D_{\varepsilon}$ is a weakly closed set in $L^{2}(0, T ; H)$ since it is closed and convex. Note that, we have

$$
\begin{aligned}
A \dot{u}_{n}(t)+B u_{n}\left(\theta_{n}(t)\right) & \in C\left(\eta_{n}(t), u\left(\theta_{n}(t)\right)\right) \\
& \subset C(t, u(t))+\left(\left|v\left(\eta_{n}(t)\right)-v(t)\right|+L\left\|u\left(\theta_{n}(t)\right)-u(t)\right\|\right) \mathbb{B} .
\end{aligned}
$$

Since $\left\|u\left(\theta_{n}(t)\right)-u(t)\right\| \rightarrow 0$ and $\left|v\left(\eta_{n}(t)\right)-v(t)\right| \rightarrow 0$ as $n \rightarrow+\infty$, one deduces that $\dot{u}_{n} \in D_{\varepsilon}$ for $n$ large enough. Thus $\dot{u} \in D_{\varepsilon}$ since $\dot{u}_{n}$ converges weakly to $\dot{u}$ in $L^{2}(0, T ; H)$. As a result, we obtain that

$$
A \dot{u}(t)+B u(t) \subset C(t, u(t))+\varepsilon \mathbb{B} \text { for every } \varepsilon>0 \text {. }
$$

Since $C(t, u(t))$ is closed and convex, we get $A \dot{u}(t)+B u(t) \in C(t, u(t))$. Now it remains to prove that

$$
\dot{u}(t) \in-N_{C(t, u(t))}(A \dot{u}(t)+B u(t)) \text { a.e. } t \in[0, T] .
$$


Given $v \in C(t, u(t))$, since $C(t, u(t)) \subset C\left(\eta_{n}(t), u\left(\theta_{n}(t)\right)\right)+\left(\left|v\left(\eta_{n}(t)\right)-v(t)\right|+\right.$ $\left.L\left\|u\left(\theta_{n}(t)\right)-u(t)\right\|\right) \mathbb{B}$, we can find some $v^{\prime} \in C\left(\eta_{n}(t), u\left(\theta_{n}(t)\right)\right)$ such that $\left\|v-v^{\prime}\right\| \leq$ $\left|v\left(\eta_{n}(t)\right)-v(t)\right|+L\left\|u\left(\theta_{n}(t)\right)-u(t)\right\|$. From (22), we have

$$
\dot{u}_{n}(t) \in-N_{C\left(\eta_{n}(t), u_{n}\left(\theta_{n}(t)\right)\right)}\left(A \dot{u}_{n}(t)+B u_{n}\left(\theta_{n}(t)\right)\right) \text { a.e. } t \in[0, T]
$$

which implies that

$$
\left\langle-\dot{u}_{n}(t), v^{\prime}-A \dot{u}_{n}(t)-B u_{n}\left(\theta_{n}(t)\right)\right\rangle \leq 0
$$

Thus

$$
\left\langle-\dot{u}_{n}(t), v-A \dot{u}_{n}(t)-B u_{n}(t)\right\rangle \leq\left\langle-\dot{u}_{n}(t), v-v^{\prime}-B u_{n}(t)+B u_{n}\left(\theta_{n}(t)\right)\right\rangle \leq \alpha_{n}(t),
$$

where

$$
\begin{aligned}
\alpha_{n}(t)= & M\left(\left|v\left(\eta_{n}(t)\right)-v(t)\right|+L\left\|u\left(\theta_{n}(t)\right)-u(t)\right\|\right. \\
& \left.+\|B\| M\left|\theta_{n}(t)-t\right|\right) \rightarrow 0 \text { as } n \rightarrow+\infty .
\end{aligned}
$$

Since it is true for all $v \in C(t, u(t))$, we deduce that

$$
\sigma\left(C(t, u(t)),-\dot{u}_{n}(t)\right)+\left\langle\dot{u}_{n}(t), A \dot{u}_{n}(t)+B u_{n}(t)\right\rangle \leq \alpha_{n}(t) .
$$

Integrating both sides of the last inequality from 0 to $T$ and taking the lower limit, one obtains

$$
\liminf _{n \rightarrow \infty} \int_{0}^{T}\left\{\sigma\left(C(t, u(t)),-\dot{u}_{n}(t)\right)+\left\langle\dot{u}_{n}(t), A \dot{u}_{n}(t)+B u_{n}(t)\right\rangle\right\} \mathrm{d} t \leq 0
$$

Let $D(t)=C(t, u(t))$ for each $t$ then $D$ has nonempty, closed convex values and varies in an absolutely continuous way. Recall that the convex mapping $x \mapsto \int_{0}^{T} \sigma(D(t), x(t)) \mathrm{d} t$ is also weakly lower semicontinuous on $L^{2}(0, T ; H)$ ([19], see also [6]). Therefore,

$$
\int_{0}^{T} \sigma(D(t),-\dot{u}(t)) \mathrm{d} t \leq \liminf _{n \rightarrow \infty} \int_{0}^{T} \sigma\left(D(t),-\dot{u}_{n}(t)\right) \mathrm{d} t .
$$

In addition, the function $x \mapsto \int_{0}^{T}\langle x(t), A x(t)\rangle \mathrm{d} t$ is also weakly lower semicontinuous on $L^{2}(0, T ; X)$ and $\dot{u}_{n}$ converges weakly to $\dot{u}$ in $L^{2}(0, T ; X)$. Thus, we have

$$
\int_{0}^{T}\langle\dot{u}(t), A \dot{u}(t)\rangle \mathrm{d} t \leq \liminf _{n \rightarrow \infty} \int_{0}^{T}\left\langle\dot{u}_{n}(t), A \dot{u}_{n}(t)\right\rangle
$$


We note that $B=\nabla \varphi_{B}$ where the function $\varphi_{B}(x):=\frac{1}{2}\langle B x, x\rangle$ is convex and continuous. Therefore, one has

$$
\begin{aligned}
\int_{0}^{T}\langle\dot{u}(t), B u(t)\rangle \mathrm{d} t & =\int_{0}^{T} \frac{\mathrm{d}}{\mathrm{d} t} \varphi_{B}(u(t)) \mathrm{d} t=\varphi_{B}(u(T))-\varphi_{B}(u(0)) \\
& =\lim _{n \rightarrow+\infty}\left(\varphi_{B}\left(u_{n}(T)\right)-\varphi_{B}\left(u_{n}(0)\right)\right) \\
& =\lim _{n \rightarrow+\infty} \int_{0}^{T} \frac{\mathrm{d}}{\mathrm{d} t} \varphi_{B}\left(u_{n}(t)\right) \mathrm{d} t \\
& =\lim _{n \rightarrow+\infty} \int_{0}^{T}\left\langle\dot{u}_{n}(t), B u_{n}(t)\right\rangle \mathrm{d} t
\end{aligned}
$$

From (32), (33), (34), (35), we obtain that

$$
\int_{0}^{T} \sigma(D(t),-\dot{u}(t))+\langle\dot{u}(t), A \dot{u}(t)+B u(t)\rangle \mathrm{d} t \leq 0 .
$$

Since $A \dot{u}(t)+B u(t) \in D(t)$ for almost $t \in[0, T]$, we have

$$
\sigma(D(t),-\dot{u}(t))+\langle\dot{u}(t), A \dot{u}(t)+B u(t)\rangle \geq 0, \quad \text { a.e. } t \in[0, T]
$$

From (36) and (37), one gets

$$
\sigma(D(t),-\dot{u}(t))+\langle\dot{u}(t), A \dot{u}(t)+B u(t)\rangle=0, \quad \text { a.e. } t \in[0, T],
$$

or equivalently,

$$
\dot{u}(t) \in-N_{D(t)}(A \dot{u}(t)+B u(t)), \quad \text { a.e. } t \in[0, T] .
$$

Therefore, $u(\cdot)$ is a solution of (11).

d) Uniqueness: Let $u_{1}(\cdot), u_{2}(\cdot)$ be two solutions of (11) with the same initial condition $u_{1}(0)=u_{2}(0)=u_{0}$. First, we prove that $\dot{u}_{i}(\cdot)$ is uniformly bounded, $i=1,2$. Indeed, since $\dot{u}_{i}(t) \in-N_{C\left(t, u_{i}(t)\right)}\left(A \dot{u}_{i}(t)+B u_{i}(t)\right)$, one has

$$
\left.\left\langle\dot{u}_{i}(t), y-A \dot{u}_{i}(t)-B u_{i}(t)\right)\right\rangle \geq 0, \forall y \in C\left(t, u_{i}(t)\right) \text {, a.e. } t \in[0, T]
$$

which implies that

$$
\left.\beta\left\|\dot{u}_{i}(t)\right\|^{2} \leq\left\langle\dot{u}_{i}(t), A \dot{u}_{i}(t)\right\rangle \leq\left\|\dot{u}_{i}(t)\right\| \| y-B u_{i}(t)\right) \| .
$$

Lemma 3.5 allows us to choose some $y_{t} \in C\left(t, u_{i}(t)\right)$ such that $\left\|y_{t}\right\| \leq c+L \| u_{i}(t)-$ $u_{0} \|$. Combining with (39), we deduce that

$$
\left.\left\|\dot{u}_{i}(t)\right\| \leq \frac{1}{\beta} \| y_{t}-B u_{i}(t)\right) \| \leq \frac{1}{\beta}\left[(L+\|B\|) \int_{0}^{t}\left\|\dot{u}_{i}(s)\right\| \mathrm{d} s+c+(L+\|B\|)\left\|u_{0}\right\|\right] .
$$


Using Gronwall's inequality, one obtains that

$$
\left\|\dot{u}_{i}(t)\right\| \leq \frac{1}{\beta}\left[(L+\|B\|)\left(c+(L+\|B\|)\left\|u_{0}\right\|\right) \exp \left(\frac{(L+\|B\|) T}{\beta}\right) .\right.
$$

Therefore, $u_{i}(t)$ and $A \dot{u}_{i}(t)+B u_{i}(t)$ are uniformly bounded by some $R>0$. From the hypomonotonicity-like of the normal cone of $C$, there exists $\tilde{L} \geq 0$ such that

$$
\begin{aligned}
& \left\langle\dot{u}_{1}(t)-\dot{u}_{2}(t), A \dot{u}_{1}(t)+B u_{1}(t)-A \dot{u}_{2}(t)-B u_{2}(t)\right\rangle \\
\leq & \tilde{L}\left\|u_{1}(t)-u_{2}(t)\right\|\left\|\dot{u}_{1}(t)-\dot{u}_{2}(t)\right\| \quad \text { a.e. } t \in[0, T] .
\end{aligned}
$$

Using the coerciveness of $A$, the last inequality implies that

$$
\begin{aligned}
& \frac{\beta}{2}\left\|\dot{u}_{1}(t)-\dot{u}_{2}(t)\right\|^{2} \\
\leq & (\tilde{L}+\|B\|)\left\|u_{1}(t)-u_{2}(t)\right\|\left\|\dot{u}_{1}(t)-\dot{u}_{2}(t)\right\|-\frac{\beta}{2}\left\|\dot{u}_{1}(t)-\dot{u}_{2}(t)\right\|^{2} \\
\leq & \frac{\tilde{L}_{1}^{2}}{2 \beta}\left\|u_{1}(t)-u_{2}(t)\right\|^{2} \text { where } \tilde{L}_{1}:=\tilde{L}+\|B\| .
\end{aligned}
$$

Hence

$$
\left\|\dot{u}_{1}(t)-\dot{u}_{2}(t)\right\| \leq \frac{\tilde{L}_{1}}{\beta}\left\|u_{1}(t)-u_{2}(t)\right\|
$$

From the Gronwall's inequality, we obtain that

$$
\left\|u_{1}(t)-u_{2}(t)\right\|^{2} \leq\left\|u_{1}(0)-u_{2}(0)\right\|^{2} \exp \left(\frac{\tilde{L}_{1} t}{\beta}\right)=0 \text { for all } t \in[0, T]
$$

Consequently, one has $u_{1} \equiv u_{2}$ and the uniqueness of solutions is obtained.

Remark 3.2 (i) The existence and uniqueness result in Theorem 3.1 can be generalized similarly for reflexive Banach spaces without any difficulty. In this case, the set-valued mapping $C:[0, T] \times X \rightrightarrows X^{*}$ and the linear operators $A, B: X \rightarrow X^{*}$, where $X$ is a reflexive Banach space with its dual $X^{*}$.

(ii) If $H \equiv \mathbb{R}^{n}$ or if some compactness assumption is supposed, then Assumption 2 and the Cauchy convergence can be skipped since in these cases, the boundedness of the iterates is sufficient to obtain the strong convergence of certain subsequence of the approximate solutions.

(iii) Although our assumptions are more general than the ones used in [13] (we consider the state-dependent case without using the additional initial viability condition), we even get the strong convergence of the approximate solutions comparing with the weak convergence of a sub-approximate solutions obtained in [13]. The coerciveness of $A$ plays an essential role in the Cauchy property of the approximate solutions. 


\section{Application to Quasistatic Frictional Contact Problem}

Let us consider the following evolution variational inequality

Find $u:[0, T] \rightarrow H$ such that $\dot{u}(t) \in \mathcal{K}$ a.e. $t \in[0, T]$ and $\forall v \in \mathcal{K}$ $a(\dot{u}(t), v-\dot{u}(t))+b(u(t), v-\dot{u}(t))+j(v)-j(\dot{u}(t)) \geq\langle f(t, u(t)), v-\dot{u}(t)\rangle$. $u(0)=u_{0} \in H$.

Suppose that the following assumptions hold:

$(\mathcal{A} 1) \mathcal{K} \subset H$ is a nonempty, closed and convex cone.

$(\mathcal{A} 2) a(\cdot, \cdot), b(\cdot, \cdot): H \times H \rightarrow \mathbb{R}$ are two real continuous bilinear and symmetric forms satisfying for all $x \in H$

$$
a(x, x) \geq \beta\|x\|^{2}, \quad \text { and } \quad b(x, x) \geq 0 \text { for some } \beta>0 .
$$

$(\mathcal{A} 3) j: \mathcal{K} \rightarrow \mathbb{R}$ is convex, positively homogeneous of degree 1 (i.e., $j(\lambda x)=$ $\lambda j(x), \forall \lambda>0)$ and Lipschitz continuous with $j(0)=0$.

(A4) $f:[0, T] \times H \rightarrow H$ satisfies

$$
\|f(t, u)-f(s, w)\| \leq|v(t)-v(s)|+L\|u-w\| \quad \forall t, s \in[0, T] \text { and } u, w \in H,
$$

for some $L \geq 0$ and some absolutely continuous function $v:[0, T] \rightarrow \mathbb{R}$.

Now, we define the functional $J: H \rightarrow \mathbb{R} \cup\{+\infty\}$ as follows:

$$
J(x)= \begin{cases}j(x), & \text { if } x \in \mathcal{K}, \\ +\infty, & \text { otherwise }\end{cases}
$$

Then $J$ is proper, positively homogeneous of degree 1 , convex, and lower semicontinuous with $J(0)=0$. Let $A, B: H \rightarrow H$ be the linear bounded and symmetric operators such that

$$
\langle A u, v\rangle=a(u, v), \quad\langle B u, v\rangle=b(u, v) .
$$

Then (42) becomes

Find $u:[0, T] \rightarrow H$ such that for a.e. $t \in[0, T]$ and $\forall v \in H$

$\langle A \dot{u}(t), v-\dot{u}(t)\rangle+\langle B u(t), v-\dot{u}(t)\rangle+J(v)-J(\dot{u}(t)) \geq\langle f(t, u(t)), v-\dot{u}(t)\rangle$, $u(0)=u_{0} \in H$,

which is equivalent to the following differential inclusion:

$$
\begin{aligned}
& f(t, u(t))-A \dot{u}(t)-B u(t) \in \partial J(\dot{u}(t)) \forall v \in H, \\
& u(0)=u_{0} \in H
\end{aligned}
$$

Proposition 4.1 Let Assumptions $(\mathcal{A} 1)-(\mathcal{A} 4)$ satisfied. Then for each $u_{0} \in H$, problem (42) has a unique solution. 
Proof First we note that the problem (42) is equivalent to (45) and (45) can be rewritten into the form of (11). Indeed, since $J$ is proper convex lower semicontinuous, positively homogeneous of degree 1 with $J(0)=0$, it is easy to see that

$$
J(y)=\sigma(C, y)=I_{C}^{*}(y)
$$

where $C=\partial J(0)$ is a closed, convex set. Thus

$$
y \in \partial J(w) \Leftrightarrow w \in \partial J^{*}(y)=\partial I_{C}(y)=N_{C}(y) .
$$

Consequently, one has

$$
\begin{aligned}
& f(t, u(t))-A \dot{u}(t)-B u(t) \in \partial J(\dot{u}(t)) \\
\Leftrightarrow & \dot{u}(t) \in N_{C}(f(t, u(t))-A \dot{u}(t)-B u(t))=N_{C-f(t, u(t))}(-A \dot{u}(t)-B u(t)) \\
\Leftrightarrow & \dot{u}(t) \in-\mathrm{N}_{C(t, u(t))}(A \dot{u}(t)+B u(t)),
\end{aligned}
$$

where $C(t, u):=f(t, u)-C=f(t, u)-\partial J(0)$, which is a particular case of Lemma 3.1. Therefore, all assumptions of Theorem 3.1 are satisfied and the conclusion follows.

Now we give an example for the quasistatic frictional contact problem involving viscoelastic materials with short memory under state-dependent perturbation forces.

Example 4.1 The physical setting consists of a deformable body $\Omega \subset \mathbb{R}^{d}(d=2,3)$ which is in contact with a rigid foundation. The boundary $\Gamma=\partial \Omega$ is supposed to be piecewise smooth composed of three parts:

$\Gamma=\Gamma_{U} \cup \Gamma_{N} \cup \Gamma_{C}$, with $\Gamma_{U} \cap \Gamma_{N}=\Gamma_{U} \cap \Gamma_{C}=\Gamma_{N} \cap \Gamma_{C}=\emptyset$ (see Fig. 1). On the boundary $\Gamma_{U}$, the body is assumed to be fixed, which means that the displacement is given and on $\Gamma_{C}$ the body is in bilateral frictional contact with a foundation, i.e., there is no loss of contact between the body and the foundation. The tractions act on $\Gamma_{N}$. In the quasistatic case, where all acceleration effects are neglected, the equilibrium of the body in this physical setting is based on the following constitutive law and boundary conditions: find a displacement field $u:[0, T] \times \Omega \rightarrow \mathbb{R}^{d}$ and stress field such that:

$$
\begin{aligned}
& \left.-\frac{\partial}{\partial t} \frac{\partial}{\partial x_{j}} a_{i j k h} \varepsilon_{k h}(u)-\frac{\partial}{\partial x_{j}} b_{i j k h} \varepsilon_{k h}(u)=\mathbf{f}_{\mathbf{0} i}(t, u), \text { in }\right] 0, T[\times \Omega \\
& \left.u_{i}=0 \text { on }\right] 0, T\left[\times \Gamma_{U}\right. \\
& \left.\sigma v=\mathbf{f}_{\mathbf{2}} \text { on }\right] 0, T\left[\times \Gamma_{N}\right. \\
& \left|\sigma_{\tau}\right| \leq g \text { on } \Gamma_{C}, u_{v}=0 \text { on } \Gamma_{C} \\
& \left|\sigma_{\tau}\right|<g \Longrightarrow \frac{\partial u_{\tau}}{\partial t}=0,\left|\sigma_{\tau}\right|=g \Longrightarrow \exists \lambda>0 \text { such that } \frac{\partial u_{\tau}}{\partial t}=-\lambda \sigma_{\tau} \\
& u(0, x)=u_{0}(x), x \in \Omega .
\end{aligned}
$$

In the equilibrium Eq. (46), $\mathbf{f}_{\mathbf{0}}(t, u)$ represents the density of a body forces, assumed to be dependent on $t$ and $u$. We suppose that the coefficients $\left(a_{i j k h}\right)$ and $\left(b_{i j k h}\right)$ have 


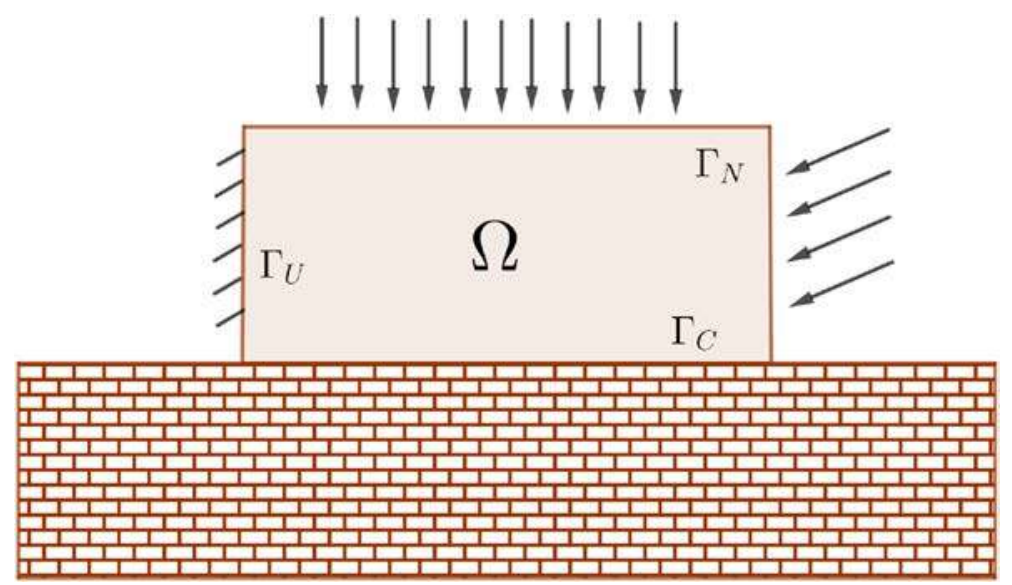

Fig. 1 A body in contact with a rigid foundation

properties of symmetry and ellipticity (see [20] for more details). Condition (48) represents the traction surface condition in which act the surface tractions density forces $\mathbf{f}_{2}$ depending on time $t$ on the boundary $\Gamma_{N}$. Using the classical notation, we denote by $\varepsilon_{i j}(u)$ and $\sigma_{i j}(u)$ the components of the strain and the stress tensor, respectively. Here ${ }^{\circ}=\left(v_{i}\right)$ denotes the outward unit normal at the boundary $\Gamma$. For any $v \in H$, we denote by $v_{\tau}$ and $v_{\nu}$ the tangential and normal components, respectively. Moreover, $\sigma_{\tau}$ and $\sigma_{\nu}$ denote the tangential and normal stress on $\Gamma$. In (49) and (50), $g \in L^{\infty}\left(\Gamma_{C}\right)$ is a nonnegative function representing a friction bound.

For the variational formulation, we introduce the following notations:

$$
\begin{aligned}
& H=\left(H^{1}(\Omega)\right)^{3} \\
& \mathcal{K}=\left\{v \in\left(H^{1}(\Omega)\right)^{3}: v=0 \text { on } \Gamma_{U} \text { and } v_{v}=0 \text { on } \Gamma_{C}\right\}, \\
& a(u, v)=\int_{\Omega} a_{i j k h} \varepsilon_{k h}(u) \varepsilon_{i j}(v) \mathrm{d} x, u, v \in H \\
& b(u, v)=\int_{\Omega} b_{i j k h} \varepsilon_{k h}(u) \varepsilon_{i j}(v) \mathrm{d} x, u, v \in H \\
& J(v)=\int_{\Gamma_{C}} g\left|v_{\tau}\right| d \Gamma, v \in H \\
& \langle f(t, u), v\rangle=\int_{\Omega} \mathbf{f}_{\mathbf{0}}(t, u) v \mathrm{~d} x+\int_{\Gamma_{N}} \mathbf{f}_{2}(t) v d \Gamma .
\end{aligned}
$$

Using standard arguments, we are led to the following variational formulation, which consists of finding $u(t)$ such that

Find $u:[0, T] \rightarrow H$ such that $\dot{u}(t) \in \mathcal{K}$ a.e. $t \in[0, T]$ and $\forall v \in \mathcal{K}$

$a(\dot{u}(t), v-\dot{u}(t))+b(u(t), v-\dot{u}(t))+J(v)-J(\dot{u}(t)) \geq\langle f(t, u(t)), v-\dot{u}(t)\rangle$, $u(0)=u_{0} \in H$. 
For more details about the variational formulation of problem (46)-(51), we refer to [20-22] for more details. We recall that if $\Omega$ is a bounded open set with a piecewise smooth boundary $\Gamma$, then the following Korn's inequality holds:

$$
\int_{\Omega} \varepsilon_{i j}(v) \varepsilon_{i j}(v) \mathrm{d} x+\int_{\Omega} v_{i} v_{i} \mathrm{~d} x \geq c\|v\|_{H}^{2}, \forall v \in H,
$$

where $c>0$ is a constant depending only on $\Omega$. Hence, the coercivity of the bilinear forms $a$ and $b$ is a consequence of the Korn's inequality. The symmetry of $a$ and $b$ follows also from the symmetry of the corresponding elasticity coefficients in (46). We suppose that $(t, u) \mapsto f_{0}(t, u)$ is absolutely continuous with respect to the time $t$ and Lipschitz continuous with respect to $u$ and that $t \mapsto f_{2}(t)$ is absolutely continuous. The following result is a consequence of Proposition 4.1.

Corollary 4.1 Under the above assumptions, for each $u_{0} \in H$, problem (58) has a unique solution which is Lipschitz continuous.

\section{Conclusions}

In this paper, we study the existence and the uniqueness of solution for a class of state-dependent implicit sweeping process in Hilbert spaces without any compactness assumption. The new notion, introduced in this paper, of hypomonotonicity-like of the normal cone to the moving set turns out to be a fundamental property, which is satisfied in many concrete situations. By using an implicit time discretization and a Cauchy technique, we obtain the strong convergence of approximate functions to the unique solution, which is an important property in view of the construction of adequate numerical algorithms. The equivalence between such implicit state-dependent sweeping processes and quasistatic evolution quasi-variational inequalities is also shown. The theoretical result is applied to analyze the state-dependent quasistatic frictional contact problem in nonsmooth mechanics. Many other open questions need further investigations. The numerical analysis of the quasistatic evolution quasi-variational inequalities using the discretization scheme studied in this paper could be also of great interest. Quasi-variational inequalities are also used to formulate many other problems in many areas such as for instance traffic transportation, Nash equilibrium in game theory and impulse control problems. It would be interesting to investigate other applications in this context. This is out of the scope of the current version and will be carried out in a future study. We expect that the ideas and tools developed in this paper will be applied in the same manner to other fields, where problems can be modeled as state-dependent implicit sweeping process with constrained velocity.

\section{References}

1. Moreau, J.J.: Sur l'evolution d'un système élastoplastique. C. R. Acad. Sci. Paris Sér. A-B 273, A118A121 (1971)

2. Moreau, J.J.: Rafle par un convexe variable I, Sém. Anal. Convexe Montpellier Exposé 15 (1971) 
3. Moreau, J.J.: Rafle par un convexe variable II, Sém. Anal. Convexe Montpellier Exposé 3 (1972)

4. Addi, K., Brogliato, B., Goeleven, D.: A qualitative mathematical analysis of a class of linear variational inequalities via semi-complementarity problems. Appl. Electron. Math. Program. 126(1), 31-67 (2011)

5. Adly, S.: A Variational Approach to Nonsmooth dynamics. Applications in Unilateral Mechanics and Electronics. Springer, Cham (2017)

6. Adly, S., Haddad, T., Thibault, L.: Convex sweeping process in the framework of measure differential inclusions and evolution variational inequalities. Math. Program. Ser. B 148(1), 5-47 (2014)

7. Goeleven, D.: Complementarity and Variational Inequalities in Electronics. Mathematical Analysis and its Applications. Academic Press, London (2017)

8. Kunze, M., Marques, M.D.P.M.: An introduction to Moreau's sweeping process. In: Brogliato, B. (ed.) Impacts in Mechanical Systems. Analysis and Modelling, pp. 1-60. Springer, Berlin (2000)

9. Adly, S., Le, B.K.: Unbounded second-order state-dependent Moreau's sweeping processes in Hilbert spaces. J. Optim. Theory Appl. 169(2), 407-423 (2016)

10. Brogliato, B.: Nonsmooth Mechanics. Models, Dynamics and Control. Communications and Control Engineering Series, 3rd edn. Springer, Cham (2016)

11. Bounkhel, M., Castaing, C.: State dependent sweeping process in p-uniformly smooth and q-uniformly convex banach spaces. Set Valued Var. Anal. 20, 187-201 (2012)

12. Adly, S., Le, B.K.: On semicoercive sweeping process with velocity constraint. Optim. Lett. 12(4), 831-843 (2018)

13. Adly, S., Haddad, T.: An implicit sweeping process approach to quasistatic evolution variational inequalities. SIAM J. Math. Anal. 50(1), 761-778 (2018)

14. Sofonea, M., Matei, A.: Variational Inequalities with Applications. A Study of Antiplane Frictional Contact Problems, Advances in Mechanics and Mathematics, vol. 18. Springer, New York (2009)

15. Clarke, F.: Functional Analysis. Calculus of Variations and Optimal Control. Springer, London (2013)

16. Brogliato, B., Goeleven, D.: Well-posedness, stability and invariance results for a class of multivalued Lur'e dynamical systems. Nonlinear Anal. Theory Methods Appl. 74, 195-212 (2011)

17. Barbu, V.: Nonlinear Differential Equations of Monotone Types in Banach Spaces. Springer, Berlin (2010)

18. Showalter, R.E.: Monotone Operators in Banach Spaces and Nonlinear Partial Differential Equations. American Mathematical Society, Providence (1997)

19. Rockafellar, R.T.: Conjugate Duality and Optimization. Conferences Board of Mathematics Sciences Series, vol. 16. SIAM, Philadelphia (1974)

20. Duvaut, D., Lions, J.L.: Inequalities in Mechanics and Physics. Springer, Berlin (1976)

21. Han, W., Sofonea, M.: Quasistatic Contact Problems in Viscoelasticity and Viscoplasticity. AMS/IP Studies in Advanced Mathematics, vol. 30. American Mathematical Society/International Press, Providence/Somerville (2002)

22. Shillor, M., Sofonea, M., Telega, J.J.: Models and Analysis of Quasistatic Contact. Springer, Berlin (2004) 